\title{
Labyrinthe
}

18 | 2004 (2)

La Recherche dans tous ses éclats

\section{Steven L. Kaplan, Le retour du bon pain}

\section{Laurent Ferri}

\section{OpenEdition}

\section{Journals}

Édition électronique

URL : http://journals.openedition.org/labyrinthe/212

DOI : $10.4000 /$ labyrinthe. 212

ISSN : 1950-6031

Éditeur

Hermann

Édition imprimée

Date de publication : 15 juillet 2004

Pagination : 37-39

Référence électronique

Laurent Ferri, «Steven L. Kaplan, Le retour du bon pain », Labyrinthe [En ligne], 18| 2004 (2), mis en ligne le 20 juin 2008, consulté le 01 mai 2019. URL : http://journals.openedition.org/labyrinthe/212 ; DOI : $10.4000 /$ labyrinthe. 212 


\section{Le Retour du bon pain de Steven L. KAPLAN*}

Laurent FERRI

laurentferri@culture.gouv.fr

Après Le Meilleur pain du monde (1996), Steven L. Kaplan poursuit sa recherche d'une identification sentimentale à la France'. Elle passe par une narration encyclopédique de l'histoire du pain, car «même si les consommateurs [en] mangent beaucoup moins, [celui-ci] continue de contenir une partie de leur identité comme Français » (p. 407). Or, dès le début des années 1970, Kaplan a pu constater que cet aliment, «dont [il avait] déjà le culte, se détériorait nettement en qualité » (p.9). Il a dès lors redouté la crise du «contrat social» séculaire qu'il résume ainsi : «Le peuple des consommateurs se soumet au fisc, à la conscription, [se montre docile] moyennant l'assurance qu'on ne le laissera pas [manquer de pain]» (p. 233)

Depuis 1945 en effet, le niveau de vie s'améliorant, la consommation recule: les ouvriers se mettent à dire "gagner son bifteck» plutôt que «gagner son pain»-1'expression renvoie alors moins aux suppliques du Pater noster qu' au souvenir de l'aliment noir, plat, lourd, et pourtant si difficile à trouver sous l'Occupation. Symbole d'un pays rural et pauvre entré dans la croissance moderne avec les Trente Glorieuses, par surcroît discrédité à l'occasion de différentes affaires d'intoxication ${ }^{2}$, le pain se détériore nettement avec le productivisme: très blanc, sur-pétri, gonflé, oxygéné, augmenté d'additifs, il devient insipide et rassis au bout de quelques heures. À ce compte, les fournils de supermarchés fabriquent un produit presque équivalent, mais moins coûteux. Ce sont, avec l'État fiscaliste, des boucs émissaires

\footnotetext{
* Éditions Perrin, 2002.

1. Présenté comme un héritier de l'école des Annales séduit par la French theory (notamment celle du linguistic turn), M. Kaplan est professeur à l'université de Cornell (Ithaca). Il vit une partie de l'année dans le XIV ${ }^{e}$ arrondissement de Paris.

2. La plus célèbre a pour cadre Pont-Saint-Esprit (Gard), entre 1951 et 1965 : elle fournira la matière du prochain livre de S. Kaplan.
} 
commodes $^{3}$. La réaction corporative, salutaire, s'opère dans les décennies 1980-1990. Toutefois, l'appui des critiques médiatiques, des sociologues, des médecins, ne permet pas de vaincre la concurrence du surgelé. On se tourne alors, comme toujours, vers l'État. Le ministre du bon pain sera un certain Jean-Pierre Raffarin, alias «Raffarine», ministre des PME, du commerce et de l'artisanat (1995-1997). Il obtient l'arrêté réservant «l'appellation et l'enseigne de boulangerie à un établissement tenu par un professionnel assurant lui-même les différentes phases de fabrication de pain ${ }^{4} \gg$. Pourtant, comme dans un rêve schumpeterien, c'est moins cette initiative gouvernementale, qui s'inscrit tout à fait dans la longue tradition d'assimilation du pain à «un service public» (p. 291), que l'apparition d'une génération d'entrepreneurs qui va permettre le retour presque partout du «bon pain» et la quasi-éviction du mauvais. Avec un plaisir évident, M. Kaplan nous présente une surprenante série de success stories à la française. Ses héros réalisent parfois la synthèse de plusieurs fantasmes très français : conciliation du terroir et de la modernité sans frontières, ascension sociale par l'assimilation... Ainsi Holder, petit-fils d'immigrés polonoukrainiens et mineurs par son père, descendant de trois générations de boulangers du Nord par sa mère, se retrouve à la tête d'une multinationale employant quatre mille personnes ( «Pau», au cœur même des hypermarchés). D'autres noms symbolisent «le métier [de boulanger] comme entreprise» sans reniement de la tradition.

Le Retour du bon pain est-il un livre d'histoire? Passé de l'Ancien Régime au temps présent, enquêtant comme un journaliste ${ }^{5}$, Steven Kaplan mélange les genres: il n'hésite pas, au détour d'un chapitre, à

3. On aurait aimé, à ce sujet, une évaluation du succès, auprès des meuniers et des boulangers, des poujadistes puis du Front national. De manière plus générale, il nous semble que l'imaginaire populiste, de droite ou de gauche, rencontre volontiers celui du pain, depuis le général Boulanger jusqu'à José Bové en passant par l'imaginaire des affameurs du peuple (le «Qu'ils mangent de la brioche!» de Marie-Antoinette ravivé en 1989 au moment du bicentenaire de la Révolution française).

4. Un dépouillement des archives de son cabinet (surtout CAC 19980121) permettrait sans doute d'étudier les probables actions de lobbying menées auprès de M. Raffarin-ministre issu du monde de l'agroalimentaire - et ayant abouti à l'arrêté du 9 mai 1995 précité.

5. Non content de parcourir toute la presse spécialisée, il a ainsi rencontré de nombreux professionnels du pain : «Partout en France, artisans, industriels, meuniers, syndicats et autres m'ont reçu et parlé ouvertement [...]. Seul Carrefour a essayé d'imposer des conditions de contrôle inadmissibles dans le cadre d'une étude comme la mienne. Ne pouvant discuter avec la direction des choix et de la politique de ce gigantesque réseau de distribution, j'ai dû mener mon enquête à la base, dans plusieurs fournils de magasin» (p. 211). 


\section{Le Retour du bon pain}

nous donner sa réponse à la question: «Comment reconnaître le bon pain?», voire à nous proposer un barème de notation. Ce qui donne au livre son épaisseur historique est le récit d'une crise faisant apparaître les trois strates temporelles chères à Fernand Braudel: la structure, la conjoncture et l'événement. En osant être écrivain, Kaplan réalise également le programme des Annales. Comme l'a rappelé un autre spécialiste des mentalités, Jonathan Dewald, «les historiens des Annales doivent être lus selon une perspective aussi bien littéraire que scientifique. Comme les écrivains de toutes catégories, ils recyclaient et transformaient les traditions intellectuelles qui les environnaient. L'intégration de figures telles que celles de Sainte-Beuve, Taine et consorts dans notre généalogie de la pratique historique peut nous aider à mettre au jour ces fonctions essentiellement artistiques qui opèrent dans l'écriture historique actuelle comme processus cherchant à donner forme au présent autant qu'à comprendre le passé $»$.

Bref, le lecteur peut s'alimenter d'histoire et d'histoires en même temps que de pain, en une promesse de surgissement du passé véritablement... proustienne. Après tout, avec sa pâte qui fermente et qui lève, sa croûte dorée, son équilibre épaisseur/émiettement, le pain ne présente-t-il pas «la structure anatomique des objets herméneutiques» dont parle le phénoménologue Jean-Pierre Richard, «éveillants pour l'imagination et la mémoire parce que suprêmement éveillés »'?

6. J. Dewald, «"À la table de Magny": XIX ${ }^{\text {th }}$ century French Men of letters and the sources of modern historical thoughts », dans The American Historical Review, octobre 2003, p. 1033. Je traduis.

7. Proust et le monde sensible, Seuil, collection «Points», 1974, p. 204 